\title{
The formation of intracellular nanoparticles correlates with cisplatin resistance
}

\author{
Meng Cao ${ }^{1,2 \dagger}$, Fangzhou Liu ${ }^{1,3,4}$, Xiquan Zhang ${ }^{1,5 \dagger}$, Ming Zheng ${ }^{5}$, Ziqi Ye ${ }^{1,6}$, Weiwei Chang ${ }^{1,5}$, Min Ji ${ }^{1}$, \\ $\mathrm{Xi} \mathrm{Zhan}^{3 *}$ and Ning $\mathrm{Gu}^{1^{*}}$
}

\begin{abstract}
Patients treated with the cisplatin often develop strong resistance to the drug after prolonged treatments, ultimately resulting in limited clinical efficacy. One of the possible mechanisms is that the internalized compound may be inactivated before getting access to the nucleus where cisplatin forms a complex with the genomic DNA and triggers a cell death program. However, the nature and intracellular fate of inactivated cisplatin is poorly illustrated. In the present study, we reported for the first time the presence of platinum nanoparticles (Pt-NPs) in the cytoplasm of cells treated with cisplatin. Further analysis also evidenced a correlation of the increased intracellular PtNPs formation with cisplatin resistance, and confirmed the process was glutathione S-transferase relevant. Our data suggest that tumor cells may develop cisplatin resistance by converting the drug into less toxic intracellular Pt-NPs, thereby impeding the drug from targeting its substrates.
\end{abstract}

\section{INTRODUCTION}

Cisplatin (cis-dichlorodiamineplatinum, CDDP or DDP) is an antineoplastic drug that is often used to treat cancers, including those derived from the head, the neck, and the lung [1-4]. Using cisplatin alone or in combination with other chemotherapeutics can effectively kill rapidly malignant cells, and has been a routine option for systematic elimination of cancerous cells following surgeries and radiotherapies [5-10]. The antineoplastic activity of cisplatin is largely attributed to its property to bind to and crosslink DNA, and to inhibit transcription and/or DNA replication, thereby triggering an apoptotic program in the targeted cells [11-14]. However, there is growing evidence for patients to develop gradually resistance to cisplatin, the concern that has drawn a great attention in clinical practices
$[15,16]$.

Cisplatin resistance may take place at different stages of chemotherapy. These include the initial contact of tumor cells with the drug, extrusion of intracellular drugs after internalization, inactivation of the drug through chemical reactions, and impairing of its ability to trigger a death signaling pathway within tumor cells. All these events would ultimately lead to increase of tumor survivals and drug tolerance $[13,17,18]$. The drug resistance that is developed before getting access to its targets is called pre-target resistance, which occurs often as the result of reduced drug accumulation [19-21]. Overexpression of glutathione S-transferase (GST), which mediates the conjugation of the reduced form of glutathione (GSH) to cisplatin and subsequent detoxification of the drug, provides an additional cause for the pre-target resistance [22-24]. However, the nature of inactivated cisplatin molecules and the fate of cisplatin within cells after inactivation remains poorly illustrated.

We noticed that cisplatin molecule contains a platinum (Pt) complex that has certain oxidability, the property that could allow it to be attacked by reducing agents in the cytoplasm and convert it into other chemical forms with a lower toxicity. Indeed, it has been reported that certain cellular substances can mediate the transformation of Pt-containing compounds into particles in live cells [25-30]. Therefore, the similar Pt particles might be also formed when cisplatin molecules interact with cytoplasm-associated reducing agents. In this study, we explored this possibility by examining cisplatin-treated tumor cells with several physical analyses, including transmission electron microscopy

\footnotetext{
${ }^{1}$ State Key Laboratory of Bioelectronics, School of Biological Science \& Medical Engineering, Southeast University, Nanjing 210096, China

${ }^{2}$ School of Public Health, Southeast University, Nanjing 210009, China

${ }^{3}$ The Center for Inflammatory and Vascular Diseases, Department of Pathology, University of Maryland School of Medicine Baltimore, Baltimore, MD 21201, USA

${ }^{4}$ Department of Head and Neck Surgery, Jiangsu Cancer Hospital, Nanjing 210009, China

${ }^{5}$ Nanjing Chia-tai Tianqing Pharmaceutical Co. Ltd, Nanjing 210038, China.

${ }^{6}$ College of Life and Environmental Science, Wenzhou University, Wenzhou 325035, China

${ }^{\dagger}$ These authors contributed equally to this work.

* Corresponding authors (emails: Guning@seu.edu.cn (Gu N); XZhan@som.umaryland.edu (Zhan X)).
} 
(TEM), dynamic light scattering (DLS) and light absorption spectrum. We found that the treated cells contained significant amounts of nanoparticle-like substances that share an atomic property with that of elemental platinum. Similar nanoparticles were also formed when cisplatin was mixed with either cell lysates or a series of reducing agents. Also, we demonstrated that Pt particles were unable to compromise the viability of tumor cells. Thus, our data suggest that conversation of cisplatin to nanoparticles by reducing agents contributes to a new mechanism for the progression of cisplatin resistances at a pre-target stage.

\section{RESULTS AND DISCUSSION}

\section{Intracellular cisplatin-related particle-like structures}

The intracellular fate of cisplatin was examined by using human HEp2 tumor cells, which have a great sensitivity to the drug. TEM revealed that many condensed particles with high contrasts were present in the cytoplasm of the cells treated with cisplatin (Fig. 1a, arrows). The detection was apparently specific for cisplatin-treated cells because these particles were not found in the cells without cisplatin treatment (Fig. 1b). Analysis of the lysates prepared from cisplatin-treated cells with TEM also displayed the presence of small particles with an average size less than $5 \mathrm{~nm}$ (Fig. 2a). To determine whether cells contained any substances that were able to induce the formation of these particles from cisplatin, cell lysates were prepared from non-treated cells and incubated with cisplatin for $12 \mathrm{~h}$ in vitro prior to TEM analysis. As shown in Fig. 2b, such cisplatin-mixed lysates had also many particles with the sizes similar to those found within the cytoplasm of cisplatin-treated cells. In contrast, no particles were visualized in a cell lysate without added cisplatin (Fig. 2e). To further confirm the nature of these particles, they were compared with $\mathrm{Pt}$ nanoparticles (Pt-NPs) that were prepared by reduction of hexachloroplatinic acid, a soluble platinum chemical, with sodium citrate [31]. These synthetic particles were found to have the sizes and shapes similar to those of the particles found either inside the cisplatin-treated cells or cell lysates mixed with cisplatin (Fig. 2c). Interestingly, when hexachloroplatinic acids were incubated with cell lysates for $12 \mathrm{~h}$, many particles were also formed though the sizes of these particles appeared smaller than those found in the cells (Fig. 2d). Taken together, these data indicate that cell lysates were sufficient to trigger the formation of platinum particles from cisplatin, and support the notion that intracellular nanoparticles were produced from the internalized cisplatin.

\section{Identification of the Pt-NPs}

It has been reported that Pt-NPs are able to aggregate with proteins, thereby yielding absorptions under ultraviolet (UV) spectrum [32]. Therefore, it is worthwhile to investigate whether the Pt-NPs formed within cells may also cluster intracellular proteins and acquire a specific absorption under UV light. Indeed, cells treated with cisplatin displayed blue fluorescence with an intensity that increased as a function of exposing time (Fig. 3a). When Pt-NPs derived from hexachloroplatinic acids were incubated with

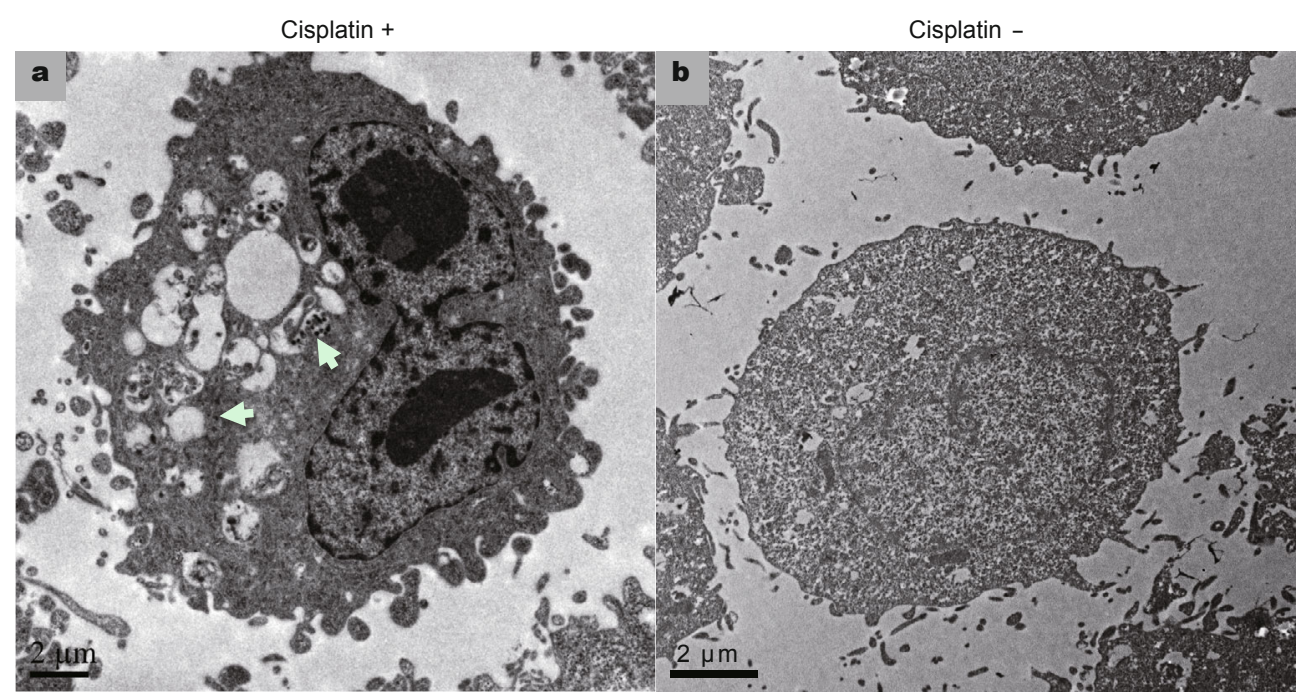

Figure 1 Cisplatin-treated cells containing particle-like structures. HEp2 cells were grown in RPMI 1640 medium supplemented with $10 \%(v / v)$ FBS and treated with cisplatin at $0.5 \mu \mathrm{g} / \mathrm{mL}$. After $24 \mathrm{~h}$, cells were fixed and analyzed by TEM. (a) Particles as pointed by arrow heads were frequently found in the cytoplasm and intracellular vesicles of a treated cell. (b) However, these particles were absent in the untreated cell. 

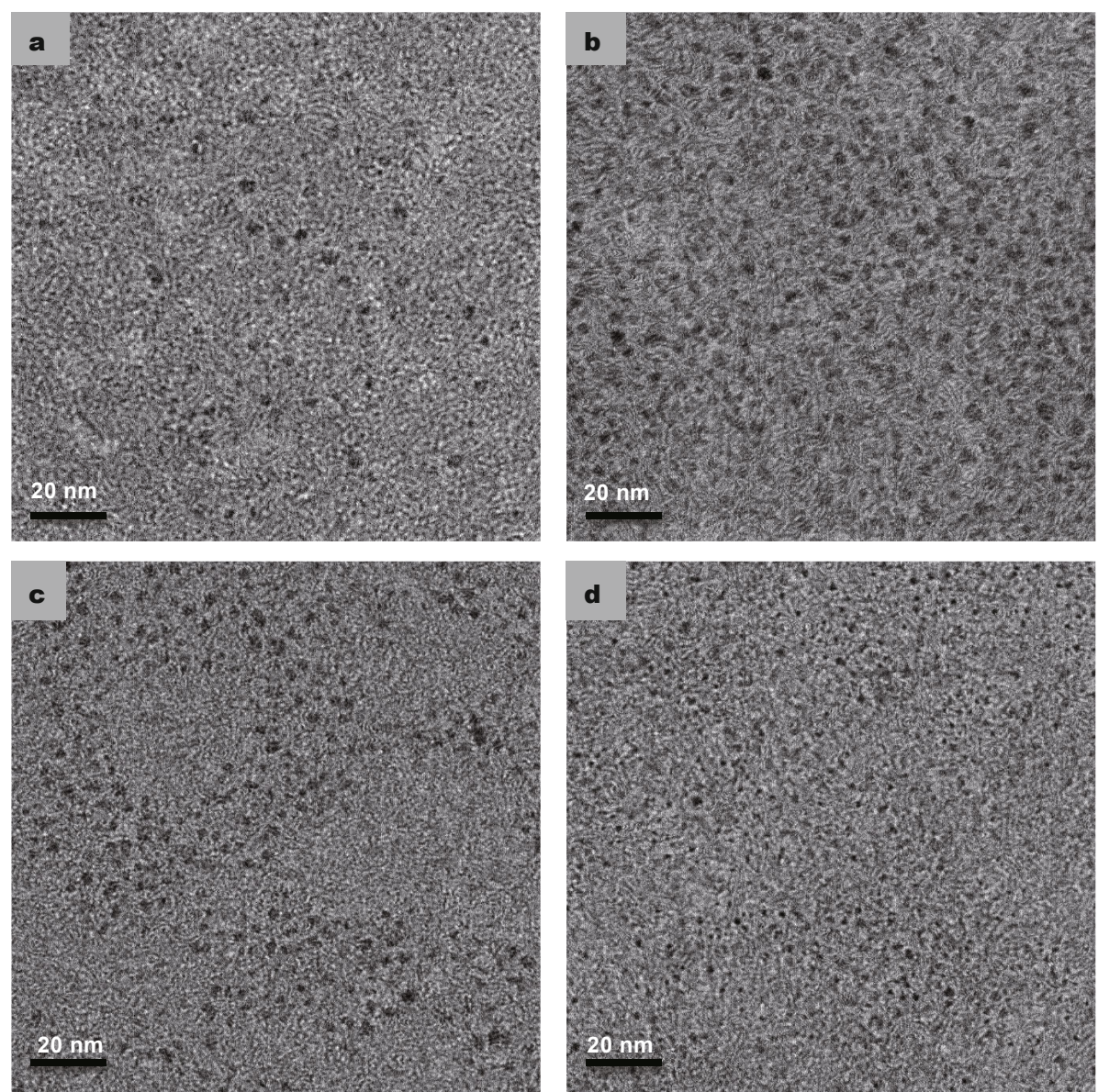

Figure 2 Formation of nanoparticles in the lysate of cisplatin-treated cells. (a) Cells were treated with $0.8 \mu \mathrm{g} / \mathrm{mL}$ cisplatin for $12 \mathrm{~h}$ and lysed subsequently by sonication. The lysate was then examined by TEM, which revealed many particles with sizes around $2-5 \mathrm{~nm}$. (b) The lysate of HEp2 cells were prepared and incubated with $0.8 \mu \mathrm{g} / \mathrm{mL}$ cisplatin for $12 \mathrm{~h}$. The cisplatin treated lysate was examined by TEM, which demonstrated the presence of numerous amounts of particles with sizes similar to those as shown in (a). (c) Synthesized nanoparticles were prepared by incubation of sodium citrate with $\mathrm{H}_{2} \mathrm{PtCl}_{6}$ and were examined by TEM. (d) Nanoparticles were also formed in cell lysates that were incubated with $1 \mu \mathrm{gg} / \mathrm{mL} \mathrm{H}_{2} \mathrm{PtCl}_{6}$ for $12 \mathrm{~h}$.

HEp2 cell lysates, they also showed increase in absorption at $220-240$ and $260-280 \mathrm{~nm}$ in a manner depending upon the dose of cell lysates (Fig. 3b), demonstrating that the increased absorbance is proportional to the formation of the complex of Pt-NPs and proteins. The emission spectrum was also analyzed, and the results showed in accordance with that of absorption (Fig. 3c).

The mixture of cisplatin with cell lysates was further analyzed by DLS, which measures the size distribution of small particles in suspension. As shown in Fig. 3d, DLS failed to give a readout of the $\mathrm{Z}$-average value with the samples containing either cisplatin alone or the cisplatin mixture with phosphate-buffered saline (PBS) (samples 4 to 6), suggesting that there were no particles generated with cisplatin dissolved in PBS. However, DLS sensed a Z-average value of about $185 \mathrm{~nm}$ with the cell lysate alone (sample 7), which could be due to some particle-like structures pres- ent in cell lysates. Under the same condition, DLS detected significantly greater Z-average values that range from 205 to $210 \mathrm{~nm}$ in samples mixed with cisplatin and different amounts of cell lysates (samples 1-3). The discrepancies $(20-25 \mathrm{~nm})$ between the mixtures and the cell lysate alone likely reflected the hydrodynamic size of particles generated from cisplatin, and were consistent with those revealed by TEM (Figs 2 and $4 a$ ), which estimated sizes of particles generally less than those obtained by DLS.

To determine whether the cisplatin-derived nanoparticles were made of elemental platinum, the lattice spacing of these nanoparticles was characterized by TEM at high resolutions. The particles in cisplatin-treated HEp2 cells after lysis had a lattice spacing of about $0.25 \mathrm{~nm}$ (Fig. 4a), which is consistent with the distance between platinum atoms in platinum crystals and in Pt-NPs [32,33]. The similar lattice spacing was also found in the particles present in the HEp2 
a
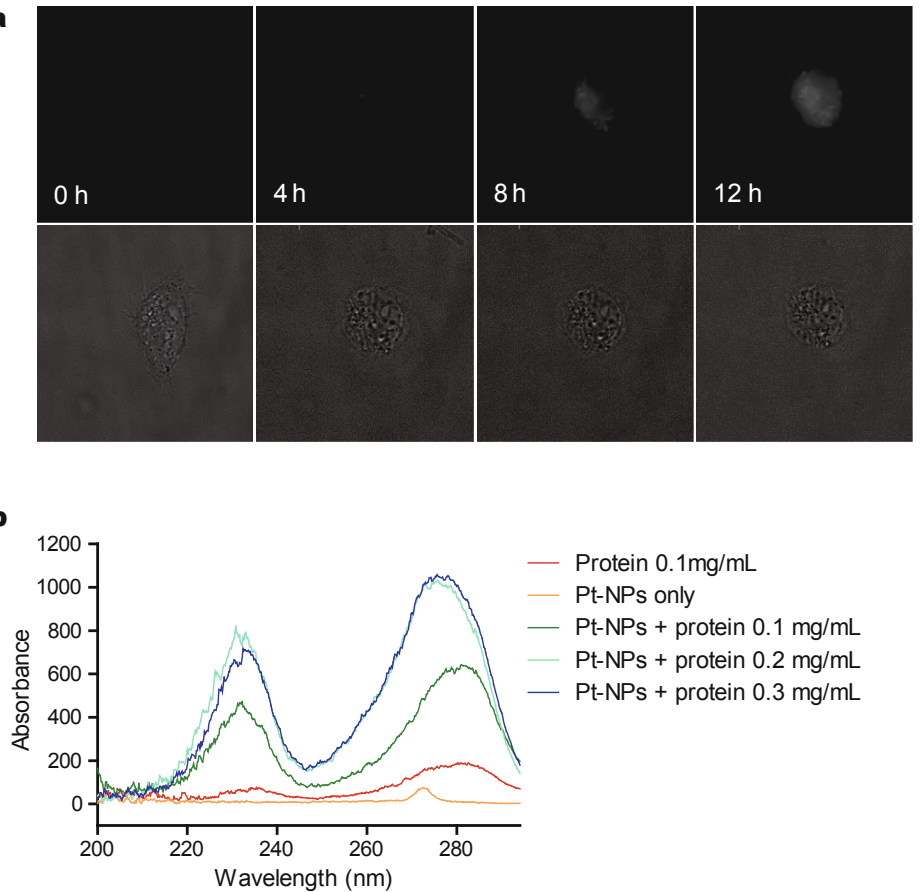

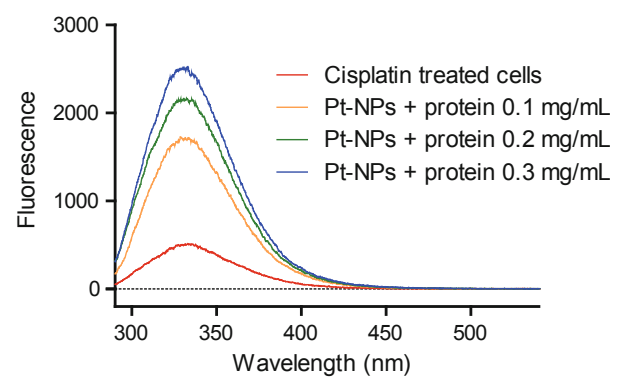

d

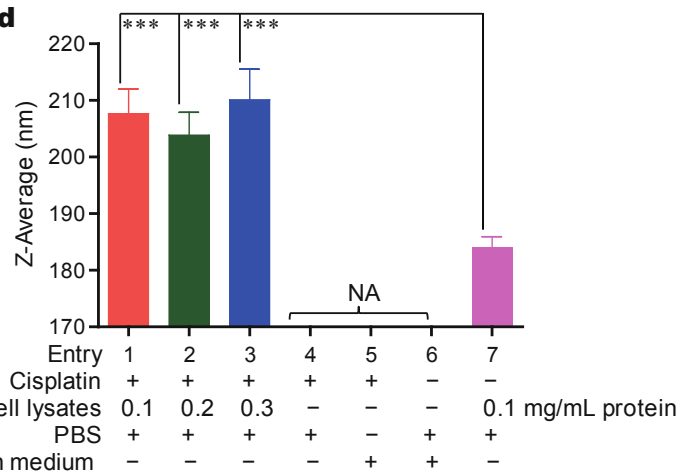

Figure 3 Spectral property and sizes of cisplatin-derived particles. (a) HEp2 cells were treated by cisplatin from 0 to $12 \mathrm{~h}$ and subsequently examined by fluorescent microscopy (top panels) or phase-contrast microscopy (bottom panels). (b) Synthesized Pt-NPs were mixed with different doses of cell lysates and subsequently examined for absorbance at UV spectra from 200 to $300 \mathrm{~nm}$. (c) The emission spectrum determination. (d) Cell lysates of different amounts of proteins were incubated with $0.25 \mathrm{mg} / \mathrm{mL}$ cisplatin for $2 \mathrm{~h}$ at $37^{\circ} \mathrm{C}$ under different conditions as indicated. The treated lysates were examined by DLS for the hydrodynamic sizes of nanoparticles. NA means not detectable.

cell lysates mixed with cisplatin (Fig. 4b), indicating that those particles are platinum nanoparticles.

To search further evidence that nanoparticles in cisplatin-treated cells were formed by a reducing reaction within cells, cisplatin was incubated for $12 \mathrm{~h}$ with several well-characterized cell-associated reducing agents, including nicotinamide adenine dinucleotide phosphate (NADPH) (Fig. 4c), GSH (Fig. 4d), ascorbic acids (Fig. 4e) and polynucleotides (Fig. 4f), respectively. In all these cases, nanoparticles were formed and displayed lattice spacing from 0.24 to $0.25 \mathrm{~nm}$, demonstrating that Pt-NPs generated by these reducing agents have the same atomic feature as those generated within cisplatin treated cells. GSH induced particles from incubation together with cisplatin were also examined under scanning electron microscope (SEM) (Supplementary information, Fig. S1a) after collected by dialysis and concentration. Energy-dispersive X-ray spectroscopy (EDS) data confirmed these particles contain platinum element (Fig. S1b).

\section{Role of Pt-NPs formation in cisplatin resistance}

Cisplatin is known to be potent in inhibiting the growth of tumor cells by triggering apoptosis [11-14]. In contrast, the cytotoxicity of Pt-NPs is quite limited [34-40]. Therefore, the conversion of internalized cisplatin molecules into Pt-NPs within cells might have contributed to the apparent drug resistance with tumor cells, which often express high levels of antioxidant enzymes such as GST [22-24]. To test this possibility, we examined $\mathrm{FaDu}$, a human pharyngeal carcinoma cell line that was reported to have a relatively higher total cellular GSH as well as a relatively high level of GST expression [23]. Indeed, FaDu cells displayed a better tolerance to cisplatin than did HEp2 cells and remained nearly $50 \%$ cell viability when treated with the drug at $16 \mu \mathrm{g} / \mathrm{mL}$ (Fig. 5b). At the same concentration, few HEp2 cells survived (Fig. 5a). For both types of cells, synthesized Pt-NPs failed to impose any significant toxicity at concentrations as high as $16 \mu \mathrm{g} / \mathrm{mL}$, confirming that Pt-NPs are generally not cytostatic. To verify whether the apparent cisplatin-tolerance of FaDu cells is correlated with the formation of Pt-NPs, the absorbance of cell lysates was measured under UV spectrum. As shown in Fig. 6a, the lysates derived from $\mathrm{FaDu}$ cells after cisplatin treatment produced a higher absorbance at $260 \mathrm{~nm}$ than did HEp2 

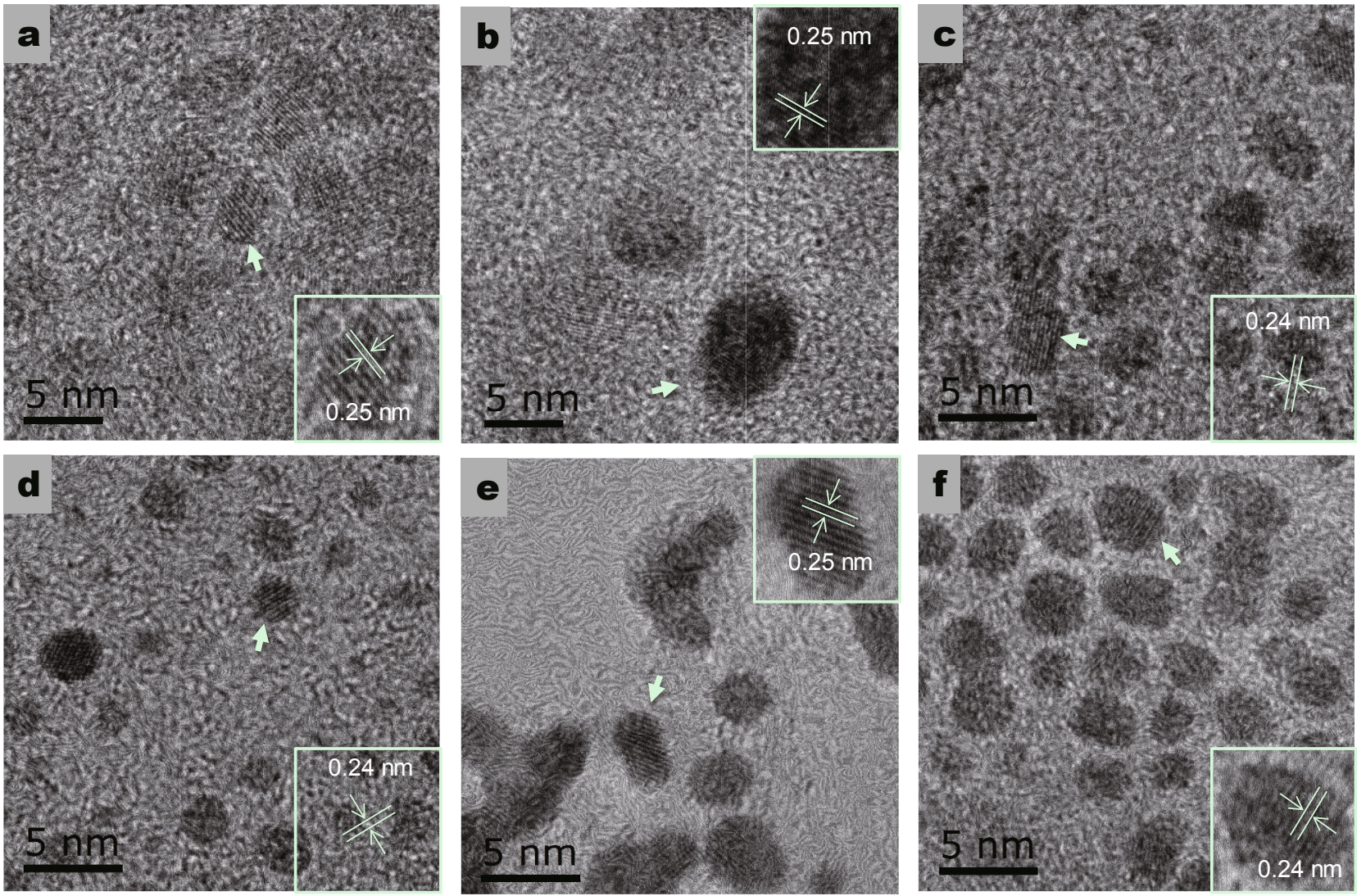

Figure 4 Characterization of lattice spacing of cisplatin-derived nanoparticles formed under different conditions. (a) The lattice spacing was estimated by TEM of the particles formed in the lysates of HEp2 cells treated with cisplatin at $0.8 \mu \mathrm{g} / \mathrm{mL}$ for $12 \mathrm{~h}$; (b) cell lysates incubated with $0.8 \mu \mathrm{g} / \mathrm{mL}$ cisplatin for $12 \mathrm{~h}$; (c) cisplatin molecules that were incubated with NADPH for $2 \mathrm{~h}$ at $37^{\circ} \mathrm{C}$; (d) cisplatin incubated with GSH for $2 \mathrm{~h}$ at $37^{\circ} \mathrm{C}$; (e) cisplatin incubated with ascorbic acid for $2 \mathrm{~h}$ at $37^{\circ} \mathrm{C}$; (f) cisplatin incubated with nucleotides for $2 \mathrm{~h}$ at $37^{\circ} \mathrm{C}$. A presentative lattice spacing for the particles in each sample was indicated.
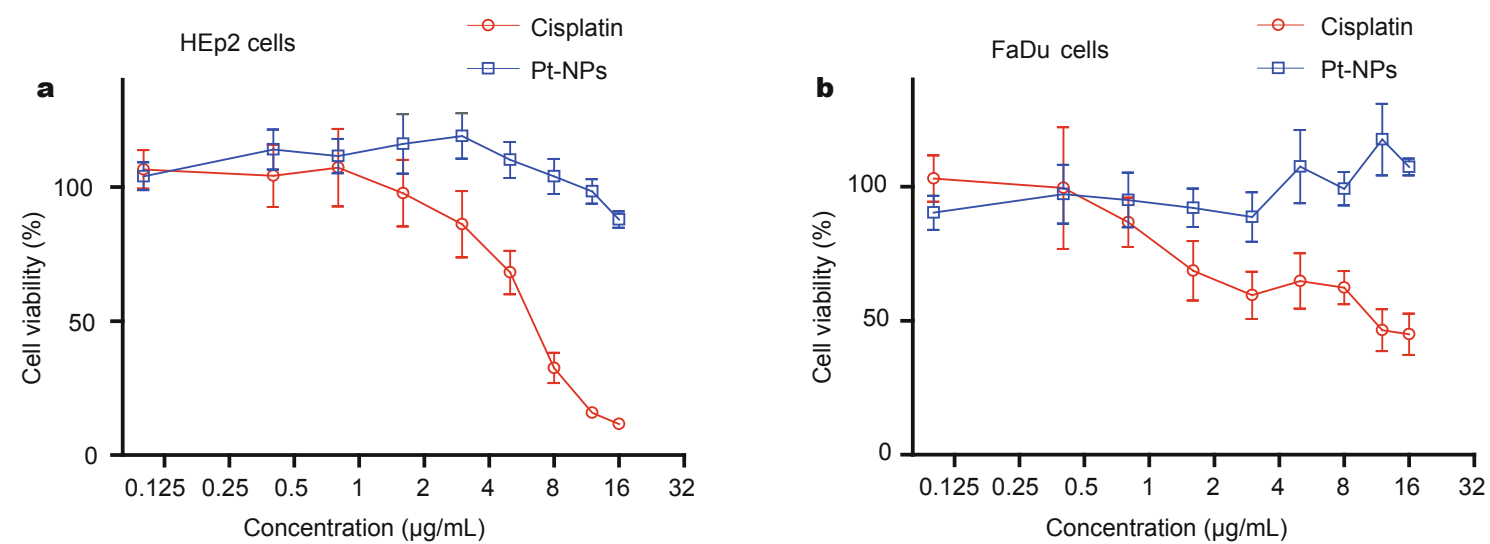

Figure 5 Pt-NPs have poor cytotoxic activity towards tumor cells. Cells were treated with either cisplatin or Pt-NPs at concentrations as indicated. After $24 \mathrm{~h}$, cell viability was analyzed by CC8 assay. (a) HEp2; (b) FaDu.

cells, suggesting a higher level of Pt-NPs present in FaDu cells than in HEp2 cells. Moreover, in consistent with the previous report that $\mathrm{FaDu}$ was compared to several other tumor cells [23], FaDu cells expressed a higher level of GST than did HEp2 cells (Fig. 6b). This indicates that the different cisplatin resistance level manifested by $\mathrm{FaDu} / \mathrm{HEp} 2$ cells may be relevant to GST mediated drug neutralization mechanism, which may be reflected as variation of cellular 


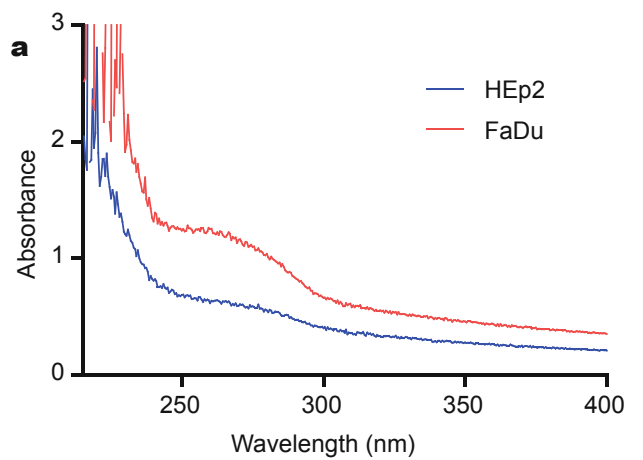

b

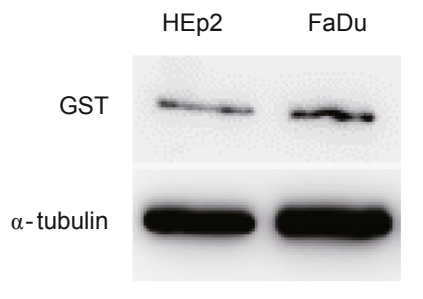

Figure 6 The formation of intracellular nanoparticles correlates with GST and cisplatin resistance. (a) HEp2 and FaDu cells were treated with $0.8 \mu \mathrm{g} / \mathrm{mL}$ cisplatin for $12 \mathrm{~h}$ and lysed. The lysates were analyzed for the UV absorbance. (b) Cells grown in the absence of cisplatin were lysed and the cell lysates were analyzed for the expression of GST by Western blot. As the loading control, the samples were also analyzed for a-tubulin expression.

Pt-NPs formation.

\section{CONCLUSION}

The pharmacological activity of cisplatin is centered on its ability to target the genomic DNA in the nucleus and subsequently trigger apoptotic programs in the treated cancer cells. Therefore, disruption of any intracellular pathways for internalized cisplatin to translocate into the nucleus would likely contribute to the progression of compromise in drug efficacy or pre-target resistances. One of the plausible mechanisms for the pre-target resistance is the increased detoxification mediated by reducing reagents such as GSH [22,23]. Part of the reason for GSH to mediate detoxification may involve its nucleophilic property that scavenges the activity of cisplatin. The conjugation of GSH to cisplatin is commonly promoted by GST, an enzyme that is frequently overexpressed in cancer cells [41-43]. Because glutathione S-conjugates are readily expelled by cells, it is thought that GSH-mediated detoxification is due to increased extrusion of conjugated cisplatin molecules. However, so far there is no clinical evidence yet for the increased release of conjugated cisplatin in resistant cells. We reported here that GSH was able to generate cisplatin-dependent nanoparticles with the similar atomic property as those found in cisplatin treated cells. Although it is unclear at present whether such conversion requires a conjugation between GSH and cisplatin, we found that several other cell-associated reducing agents, including NADPH, ascorbic acids and polynucleotides, were also able to convert cisplatin into nanoparticles similarly as was GSH. This suggests that the conjugation of cisplatin with other molecules such as GSH may not be necessary for the formation of Pt-NPs. We suggest that these particles were formed as a result of reducing reaction itself. To our knowledge, this would be the first report about the presence of Pt-NPs in cisplatin-treated cells. Since Pt-NPs have demonstrated low toxicity and antioxidants are prevalent in cells, our finding offers an alternative explanation for the high incidence of cisplatin resistance. Our finding also challenges a view for the benefit of using antioxidants in cancer therapies, and may provide an opportunity to develop new strategies to circumvent cisplatin resistance. In particular, we found that Pt-NPs formed within cisplatin-treated cells were able to produce a specific absorption under UV light (Fig. 7). Such property may be exploited to develop new nano-probes for tumor imaging, meaning that the drug resistance developed during cisplatin-based chemotherapies may be readily tracked by following the change in the optical property of treated tumors.

\section{EXPERIMENTAL SECTION}

\section{Materials}

All chemicals except for those mentioned elsewhere were purchased from Sigma. Cisplatin was from Chia-tai Tianqing Pharmaceutical. Anti-GST antibody was from Bioss. Anti-a-tubulin antibody was from KeyGen.

\section{Cell culture}

HEp2 cell line was a gift from Jiangsu Cancer Hospital. FaDu cells were from the Cell Bank of Chinese Academy of Sciences. HEp2 cells were cultured in RPMI 1640 medium (Gibco) supplemented with 10\% ( $v / v)$ fetal ovine serum (FBS), $100 \mu \mathrm{g} / \mathrm{mL}$ streptomycin and 100 units $/ \mathrm{mL}$ penicillin (Gibco) at $37^{\circ} \mathrm{C}$, under air $/ 5 \% \mathrm{CO}_{2}$ mixture atmosphere. FaDu cells were maintained in Minimum Essential Medium (MEM, Hyclone) supplemented with 10\% FBS.

\section{Electron microscopy}

Cells were treated with or without $0.5 \mu \mathrm{g} / \mathrm{mL}$ cisplatin for 


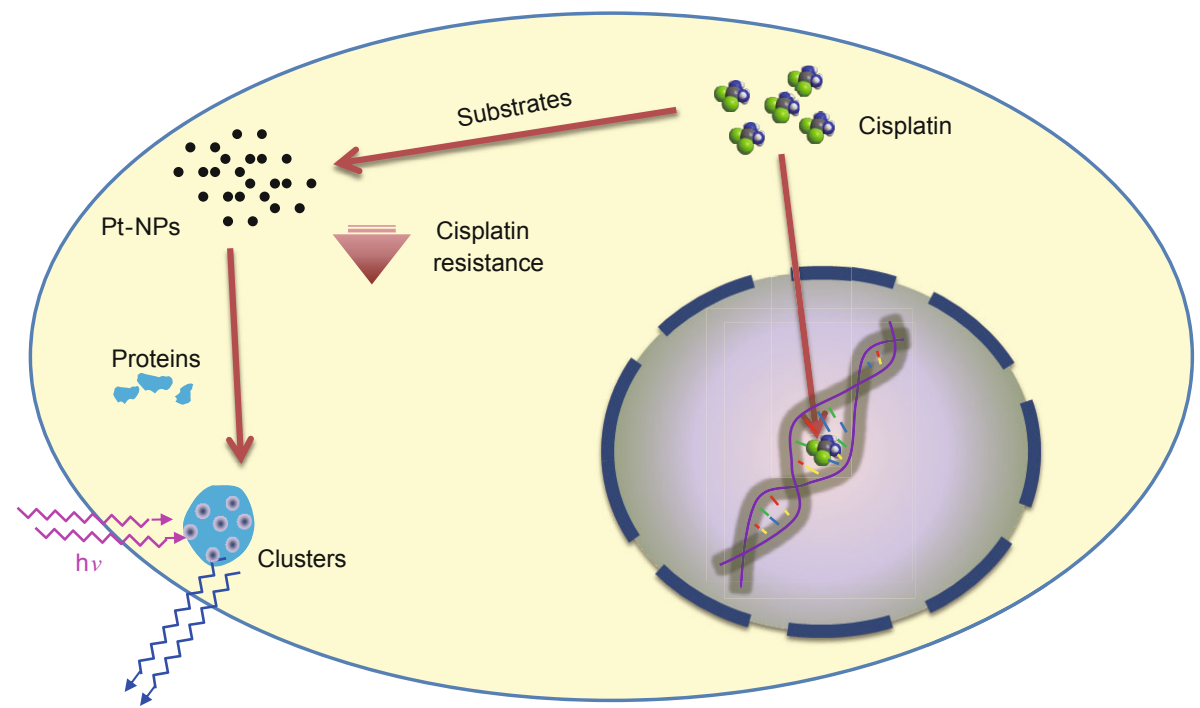

Figure 7 Schematic illustration of the possible relevance between intracellular Pt-nanoparticles formation and cisplatin resistance.

24 h. $10^{7}$ treated cells were harvested and fixed overnight in PBS containing $2.5 \%$ glutaraldehyde at $4^{\circ} \mathrm{C}$. The samples were then treated with $1 \%$ osmium tetroxide followed by dehydration in ethanol and embedding in Epon (Fluka). The fixed samples were used to generate ultrathin sections $(60-80 \mathrm{~nm})$, which were subsequently double stained with uranyl acetate and lead citrate. The stained samples (for lysates and mixtures examined under high-resolution, staining was not needed) were examined by TEM (JEOL/ JEM-2000E). For SEM and EDS, the mixture containing cisplatin and GSH was concentrated by dialysis and evaporation after incubation, and then examined under a Carl Zeiss ULTRA plus field emission scanning electron microscope with supporting by a slice of aluminum foil.

\section{Preparation of platinum nanoparticles}

Platinum nanoparticles (Pt-NPs) were prepared as described previously $[31,44]$.

\section{Spectrometry}

Cells were treated with or without cisplatin as above. The treated cells were suspended in PBS and lysed by sonication. The lysates were analyzed for absorbance at 200-400 nm using UV-3600 spectrophotometer (Shimadzu). In some experiments, cell lysates were mixed with cisplatin or synthesized Pt-NPs prior to spectrometric analysis.

\section{Immunoblotting analysis}

Cells were collected by trypsinization followed by centrifugation at $200 \times \mathrm{g}$. The pellet of $6 \times 10^{6}$ cells was lysed in $10 \mu \mathrm{L}$ $6 \times \operatorname{SDS}$ sample buffer, heated at $95^{\circ} \mathrm{C}$ for $10 \mathrm{~min}$ and sepa- rated by SDS-polyacrylamide electrophoresis at $108 \mathrm{~V}$ for $80 \mathrm{~min}$. The proteins in the gel were transferred to a polyvinylidene fluoride membrane in buffer containing $25 \mathrm{mM}$ Tris, $192 \mathrm{mM}$ glycine, and 20\% methanol. Non-specific binding sites on the membrane were blocked by incubation with $5 \%$ bovine serum albumin in TBST (100 mM Tris$\mathrm{HCl}, \mathrm{pH} 7.5,150 \mathrm{mM} \mathrm{NaCl}$, and $0.05 \%(v / v)$ Tween20) at room temperature for $30 \mathrm{~min}$. The membrane was then incubated with anti-GST or anti- $\alpha$-tubulin antibody at dilutions from 1:200 to 1:1000 in the blocking solution for overnight at $4^{\circ} \mathrm{C}$. The membrane was washed three times with TBST followed by incubating for $30 \mathrm{~min}$ with the blocking buffer containing horseradish peroxidase-conjugated secondary antibody at 1:1000 to 1:5000 dilutions. The antibody-reactive substances on the membrane were detected by Super Chemiluminescence Development Kit (KeyGen).

\section{Cell viability assay}

The cell viability was tested with Cell Counting Kit-8 (CCK-8, KeyGen). Briefly, cells were seeded in 96-well plates at the density of $3 \times 10^{3}$ cells/well and incubated in freshly prepared growth medium supplemented with $10 \%$ FBS, $100 \mu \mathrm{g} / \mathrm{mL}$ streptomycin and 100 units/mL penicillin at $37^{\circ} \mathrm{C}$ and $5 \% \mathrm{CO}_{2}$. After $24 \mathrm{~h}$, the medium was replaced with the growth medium containing a gradient cisplatin or Pt-NPs in the range from 0 to $50 \mu \mathrm{g} / \mathrm{mL}$. After incubation for 24 to $72 \mathrm{~h}$, the medium was removed to a new plate, and the cells were rinsed once with PBS and then subjected to CCK-8 analysis. 
Received 18 June 2015; accepted 15 July 2015; published online 11 August 2015

1 Prestayko AW, Daoust JC, Issell BF, et al. Cisplatin (cis-diamminedichloroplatinum-II). Cancer Treat Rev, 1979, 6: 17-39

2 Chen X, Wu Y, Dong H, et al. Platinum-based agents for individualized cancer treatment. Curr Mol Med, 2013, 13: 1603-1612

3 Maccio A, Madeddu C. Cisplatin: an old drug with a newfound efficacy-from mechanisms of action to cytotoxicity. Expert Opin Pharmaco, 2013, 14: 1839-1857

4 Stathopoulos GP. Cisplatin: process and future. J Buon, 2013, 18: 564-569

5 Perry DJ, Weltz MD, Brown AW, et al. Vinblastine, bleomycin and cisplatin for recurrent or metastatic squamous-cell carcinoma of the head and neck. Cancer, 1982, 50: 2257-2260

6 Leipzig B. Cisplatin sensitization to radiotherapy of squamous-cell carcinomas of the head and neck. Am J Surg, 1983, 146: 462-465

7 Brizel DM, Albers ME, Fisher SR, et al. Hyperfractionated irradiation with or without concurrent chemotherapy for locally advanced head and neck cancer. New Engl J Med, 1998, 338: 1798-1804

8 Bernier J, Domenge C, Ozsahin M, et al. Postoperative irradiation with or without concomitant chemotherapy for locally advanced head and neck cancer. New Engl J Med, 2004, 350: 1945-1952

9 Khuri FR, Nemunaitis J, Ganly I, et al. A controlled trial of intratumoral ONYX-015, a selectively-replicating adenovirus, in combination with cisplatin and 5-fluorouracil in patients with recurrent head and neck cancer. Nat Med, 2000, 6: 879-885

10 Posner MR, Hershock DM, Blajman CR, et al. Cisplatin and fluorouracil alone or with docetaxel in head and neck cancer. New Engl J Med, 2007, 357: 1705-1715

11 Jamieson ER, Lippard SJ. Structure, recognition, and processing of cisplatin-DNA adducts. Chem Rev, 1999, 99: 2467-2498

12 Jordan P, Carmo-Fonseca M. Molecular mechanisms involved in cisplatin cytotoxicity. Cell Mol Life Sci, 2000, 57: 1229-1235

13 Cepeda V, Fuertes MA, Castilla J, et al. Biochemical mechanisms of cisplatin cytotoxicity. Anticancer Agents Med Chem, 2007, 7: 3-18

14 Dasari S, Tchounwou PB. Cisplatin in cancer therapy: molecular mechanisms of action. Eur J Pharmacol, 2014, 740: 364-378

15 Siddik ZH. Cisplatin: mode of cytotoxic action and molecular basis of resistance. Oncogene, 2003, 22: 7265-7279

16 Shen DW, Pouliot LM, Hall MD, et al. Cisplatin resistance: a cellular self-defense mechanism resulting from multiple epigenetic and genetic changes. Pharmacol Rev, 2012, 64: 706-721

17 Galluzzi L, Senovilla L, Vitale I, et al. Molecular mechanisms of cisplatin resistance. Oncogene, 2012, 31: 1869-1883

18 Galluzzi L, Vitale I, Michels J, et al. Systems biology of cisplatin resistance: past, present and future. Cell Death Dis, 2014, 5: e1257

19 Arnesano F, Losacco M, Natile G. An updated view of cisplatin transport. Eur J Inorg Chem, 2013, 2701-2711

20 Katano K, Kondo A, Safaei R, et al. Acquisition of resistance to cisplatin is accompanied by changes in the cellular pharmacology of copper. Cancer Res, 2002, 62: 6559-6565

21 Tsai CY, Larson CA, Safaei R, et al. Molecular modulation of the copper and cisplatin transport function of CTR1 and its interaction with IRS-4. Biochem Pharmacol, 2014, 90: 379-387

22 Nishimura T, Newkirk K, Sessions RB, et al. Immunohistochemical staining for glutathione S-transferase predicts response to platinum-based chemotherapy in head and neck cancer. Clin Cancer Res, 1996, 2: 1859-1865

23 Cullen KJ, Newkirk KA, Schumaker LM, et al. Glutathione S-transferase $\pi$ amplification is associated with cisplatin resistance in head and neck squamous cell carcinoma cell lines and primary tumors. Cancer Res, 2003, 63: 8097-8102
24 Shiga H, Heath EI, Rasmussen AA, et al. Prognostic value of p53, glutathione S-transferase $\pi$, and thymidylate synthase for neoadjuvant cisplatin-based chemotherapy in head and neck cancer. Clin Cancer Res, 1999, 5: 4097-4104

25 Chiu CY, Ruan L, Huang Y. Biomolecular specificity controlled nanomaterial synthesis. Chem Soc Rev, 2013, 42: 2512-2527

26 Faramarzi MA, Sadighi A. Insights into biogenic and chemical production of inorganic nanomaterials and nanostructures. Adv Colloid Interface Sci, 2013, 189-190: 1-20

27 Konishi Y, Ohno K, Saitoh N, et al. Bioreductive deposition of platinum nanoparticles on the bacterium shewanella algae. J Biotechnol, 2007, 128: 648-653

28 Song JY, Kwon EY, Kim BS. Biological synthesis of platinum nanoparticles using Diopyros kaki leaf extract. Bioprocess Biosyst Eng, 2010, 33: 159-164

29 Venu R, Ramulu TS, Anandakumar S, et al. Bio-directed synthesis of platinum nanoparticles using aqueous honey solutions and their catalytic applications. Colloids and Surfaces A: Physicochem Eng Aspects, 2011, 384: 733-738

30 Govender Y, Riddin TL, Gericke M, et al. On the enzymatic formation of platinum nanoparticles. J Nanopart Res, 2010, 12: 261-271

31 Aika K, Ban LL, Okura I, et al. Chemisorption and catalytic activity of a set of platinum catalysts. J Res Inst Catalysis Hokkaido Univ, 1976, 24: 54-64

32 Li M, Yang DP, Wang XS, et al. Mixed protein-templated luminescent metal clusters ( $\mathrm{Au}$ and $\mathrm{Pt}$ ) for $\mathrm{H}_{2} \mathrm{O}_{2}$ sensing. Nanoscale Res Lett, 2013, 8: 182

33 Li YJ, Whyburn GP, Huang Y. Specific peptide regulated synthesis of ultrasmall platinum nanocrystals. J Am Chem Soc, 2009, 131: 15998-15999

34 Konieczny P, Goralczyk AG, Szmyd R, et al. Effects triggered by platinum nanoparticles on primary keratinocytes. Int J Nanomed, 2013, 8: 3963-3975

35 Yamagishi Y, Watari A, Hayata Y, et al. Acute and chronic nephrotoxicity of platinum nanoparticles in mice. Nanoscale Res Lett, 2013, 8: 395

36 Elder A, Yang H, Gwiazda R, et al. Testing nanomaterials of unknown toxicity: an example based on platinum nanoparticles of different shapes. Adv Mater, 2007, 19: 3124-3129

37 Asharani PV, Xinyi N, Hande MP, et al. DNA damage and p53-mediated growth arrest in human cells treated with platinum nanoparticles. Nanomedicine, 2010, 5: 51-64

38 Asharani PV, Yi LW, Gong ZY, et al. Comparison of the toxicity of silver, gold and platinum nanoparticles in developing zebrafish embryos. Nanotechnology, 2011, 5: 43-54

39 Gehrke H, Pelka J, Hartinger CG, et al. Platinum nanoparticles and their cellular uptake and DNA platination at non-cytotoxic concentrations. Arch Toxicol, 2011, 85: 799-812

40 Horie M, Kato H, Endoh S, et al. Evaluation of cellular influences of platinum nanoparticles by stable medium dispersion. Metallomics, 2011, 3: 1244-1252

41 Tsuchida S, Sato K. Glutathione transferases and cancer. Crit Rev Biochem Mol Biol, 1992, 27: 337-384

42 Hayes JD, Pulford DJ. The glutathione S-transferase supergene family: regulation of gst and the contribution of the isoenzymes to cancer chemoprotection and drug resistance. Crit Rev Biochem Mol Biol, 1995, 30: 445-600

43 Townsend DM, Tew KD. The role of glutathione-s-transferase in anti-cancer drug resistance. Oncogene, 2003, 22: 7369-7375

44 Henglein A, Ershov BG, Malow M. Absorption-spectrum and some chemical-reactions of colloidal platinum in aqueous-solution. J Phys Chem, 1995, 99: 14129-14136 
Acknowledgments This work was Funded by the National Key Basic Research Program of China (2011CB933503), the National Natural Science Foundation of China (NSFC) for Key Project of International Cooperation (61420106012), the Special Funds of National Natural Science Foundation of China For Basic Research Projects of Scientific Instruments (61127002), the Special Project on the Development of National Key Scientific Instruments and Equipment of China (2011YQ03013403), and China Postdoctoral Science Foundation funded project (2013M541592).

Author contributions Gu N, Zhan X, Ji M and Cao M conceived and designed the reported research. Liu F and Zhang X prepared the samples and performed the TEM experiments, assisted by Zheng M. Cao M, Ye Z and Chang $\mathrm{W}$ maintained the four cell lines and performed cell viability assays as well as western blot. Cao M, Chang $\mathrm{W}$ and Zheng $\mathrm{M}$ designed the particle validation experiments and performed spectrometry. Cao M, Liu F and Zhang X analyzed the data. Gu N, Zhan X, Ji M and Cao M wrote the manuscript. All authors discussed the results and commented on the manuscript.

Conflict of interest The authors declare that they have no conflict of interest.

Supplementary information Supplementary information including the SEM examination of particles generated from cisplatin is available in the online version of the paper.

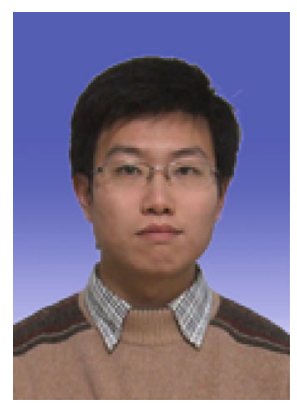

Meng Cao was born in 1983. He received his PhD degree in biomedical engineering from the School of Biological Science \& Medical Engineering, Southeast University, Nanjing, China, in 2013. Currently he is a postdoctoral fellow in Professor Ning Gu's group. His research interests mainly focus on biochemistry and anti-tumor therapeutics.

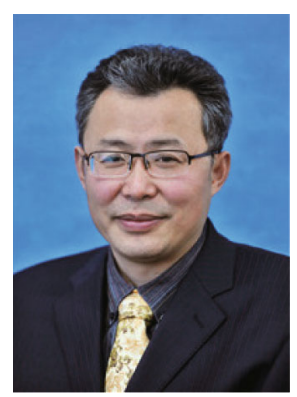

Ning Gu was born in 1964. He received his PhD degree in biomedical engineering from the Department of Biomedical Engineering, Southeast University, Nanjing, China, in 1996. Currently he is a Changiiang Scholar Professor and NSFC Outstanding Young Investigator Fund Winner at the School of Biological Science and Medical Engineering, Southeast University. He also serves as the president of Jiangsu Society of Biomedical Engineering, the director of the Research Center for Nanoscale Science and Technology of Southeast University. His research interests include biomaterials, nanobiology, medical imaging, and advanced instrument development.

中文摘要 长期使用顺铂治疗的肿瘤患者容易产生肿瘤细胞耐药性, 影响顺铂的临床治疗效果. 顺铂进入肿瘤细胞后, 在到达靶向细胞 核之前可能会失去活性, 失活后的药物将无法与DNA络合以杀伤细胞, 这是细胞产生药物耐受性的可能机制之一. 然而失活后的顺铂 在细胞内以何种形式存在这一问题尚没有得到完全阐明. 本论文研究发现, 顺铂治疗后的细胞中有铂纳米颗粒存在, 而且该类颗粒的 形成与细胞对顺铂的耐药性有一定的关系. 进一步研究发现细胞内谷胱甘肽转移酶可能在这一机制中起到了重要作用. 数据表明, 细 胞可能通过将高细胞毒性的顺铂转化为低毒性的铂纳米颗粒, 起到阻止顺铂靶向其底物的作用. 